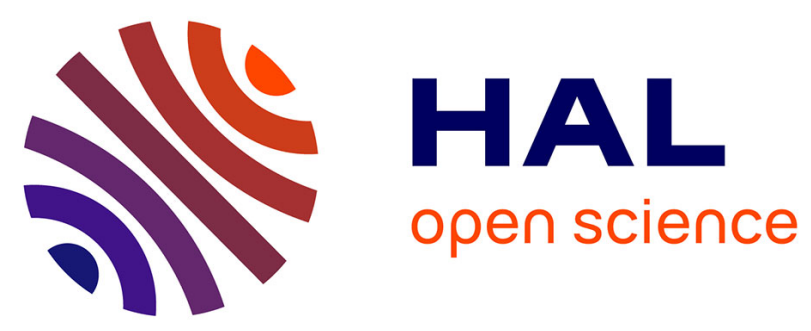

\title{
Slope and curvature of the hadronic vacuum polarization at vanishing virtuality from lattice QCD
}

Sz. Borsanyi, Z. Fodor, T. Kawanai, S. Krieg, Laurent Lellouch, R. Malak, K. Miura, K. K. Szabo, C. Torrero, B. Toth

\section{- To cite this version:}

Sz. Borsanyi, Z. Fodor, T. Kawanai, S. Krieg, Laurent Lellouch, et al.. Slope and curvature of the hadronic vacuum polarization at vanishing virtuality from lattice QCD. Physical Review D, 2017, 96 (7), pp.074507 10.1103/PhysRevD.96.074507 . hal-01477989

\section{HAL Id: hal-01477989 \\ https://hal.science/hal-01477989}

Submitted on 4 May 2018

HAL is a multi-disciplinary open access archive for the deposit and dissemination of scientific research documents, whether they are published or not. The documents may come from teaching and research institutions in France or abroad, or from public or private research centers.
L'archive ouverte pluridisciplinaire HAL, est destinée au dépôt et à la diffusion de documents scientifiques de niveau recherche, publiés ou non, émanant des établissements d'enseignement et de recherche français ou étrangers, des laboratoires publics ou privés. 


\title{
Slope and curvature of the hadronic vacuum polarization at vanishing virtuality from lattice QCD
}

\author{
Sz. Borsanyi, ${ }^{1}$ Z. Fodor, ${ }^{1,2,3}$ T. Kawanai, ${ }^{3}$ S. Krieg, ${ }^{1,3}$ L. Lellouch,${ }^{4}$ R. Malak,${ }^{4,5}$ K. Miura,${ }^{4}$ \\ K. K. Szabo, ${ }^{1,3}$ C. Torrero, ${ }^{4}$ and B. C. Toth ${ }^{1}$ \\ ${ }^{1}$ Department of Physics, Bergische Universität Wuppertal, Gaussstrasse 20, D-42119 Wuppertal, Germany \\ ${ }^{2}$ Institute for Theoretical Physics, Eötvös University, Pázmány Péter sétány 1/A, H-1117 Budapest, Hungary \\ ${ }^{3}$ Jülich Supercomputing Centre, Forschungszentrum Jülich, D-52425 Jülich, Germany \\ ${ }^{4}$ CNRS, Aix-Marseille Univ, Université de Toulon, CPT, UMR 7332, F-13288 Marseille, France \\ ${ }^{5}$ CNRS, CEA, Maison de la Simulation, USR 3441, F-91191 Gif-sur-Yvette Cedex, France
}

(Received 19 January 2017; published 24 October 2017)

\begin{abstract}
We compute the slope and curvature, at vanishing four-momentum transfer squared, of the leading order hadronic vacuum polarization function, using lattice quantum chromodynamics. Calculations are performed with $2+1+1$ flavors of staggered fermions directly at the physical values of the quark masses and in volumes of linear extent larger than $6 \mathrm{fm}$. The continuum limit is carried out using six different lattice spacings. All connected and disconnected contributions are calculated, up to and including those of the charm.
\end{abstract}

DOI: 10.1103/PhysRevD.96.074507

\section{INTRODUCTION}

The vacuum expectation value of the product of two electromagnetic currents plays an important role in physics. It describes how virtual particle fluctuations polarize the vacuum as it is traversed by a propagating photon. While the contributions associated with virtual leptons and weak bosons can be computed in perturbation theory, those of quarks require nonperturbative methods for small photon virtuality, because of the confinement of quarks within hadrons. Here we focus on the latter, known as the hadronic vacuum polarization or HVP.

The low energy behavior of the HVP is the limiting uncertainty in the standard model (SM) prediction of a number of quantities. It limits the precision with which many electroweak observables are determined [1]. It also represents the leading hadronic uncertainty in the SM prediction for the anomalous magnetic moments of leptons, $a_{\ell}$ with $\ell=e, \mu, \tau$ [2,3]. In fact, it is the limiting factor in the SM prediction [2-8] of the much debated anomalous magnetic moment of the muon that is currently measured to $0.54 \mathrm{ppm}[9]$.

Today the HVP is best determined using dispersion relations and the cross section of $e^{+} e^{-}$to hadrons or the rate of hadronic $\tau$ decays [6-8]. However, since the pioneering work of [10], lattice quantum chromodynamics (QCD) calculations of the leading order (LO) HVP contributions, $a_{\mu}^{\mathrm{LO}-\mathrm{HVP}}$, to $a_{\mu}$ have made significant progress [11-21]. Moreover, in the long run, this approach is likely to represent the most cost-effective way to increase the precision of the HVP to the levels that will soon be required by the new round of measurements of $a_{\mu}[22,23]$ and, more generally, by particle physics phenomenology.

Here we present a full lattice QCD calculation of the first two derivatives of the HVP function at zero, Euclidean virtuality. The calculation includes all contributions from $u$, $d, s$ and $c$ quarks, both in their quark-connected and quarkdisconnected configurations, in the isospin limit. As shown in [24,25], the slope of the polarization function provides an upper bound on the HVP contribution to the anomalous magnetic moment of all three leptons. It also determines the whole of $a_{\ell}^{\mathrm{LO}-\mathrm{HVP}} / m_{\ell}^{2}$ in the limit that the lepton mass, $m_{\ell}$, vanishes [24]. Moreover, together with the curvature, the slope gives $a_{\mu}^{\mathrm{LO}-\mathrm{HVP}}$ to within less than $2 \%$. This fraction, which is indicative only, derives from comparing the value of $a_{\mu}^{\mathrm{LO}-\mathrm{HVP}}$ obtained from a simple phenomenological model to that obtained using a $[1,1]$ Padé approximant to describe the virtuality dependence of the HVP function. This approximant is constructed from the slope and curvature of the HVP function at vanishing virtuality, obtained in the same model. The model combines the $e^{+} e^{-}$experimental spectrum up to the $\psi^{\prime}$ that is compiled in [26], perturbative contributions above $s=2.25 \mathrm{GeV}^{2}$ and dispersion relations. The use of Padé approximants for determining $a_{\mu}^{\mathrm{LO}-\mathrm{HVP}}$ was first proposed in [27] and was first used for a lattice QCD evaluation in [16]. Since the appearance of the present work in preprint form [28], other methods have been developed for determining $a_{\mu}^{\mathrm{LO}-\mathrm{HVP}}$ from the slope and curvature of the HVP with similar or better accuracy $[29,30]$. The first is based on approximations of the Mellin-Barnes representation of the spectral function [29] and the second on finite-energy sum rules [30].

The main challenge in calculating, on the lattice, derivatives of the HVP function at zero virtuality is the fact that it requires determining, with high precision, the dominant, and notoriously noisy, $u$ and $d$ quark contributions to the electromagnetic current correlation function at large euclidean distances. This is particularly clear when these derivatives are obtained by computing moments, in Euclidean spacetime, of the quark, electromagnetic twopoint function, as described below, around Eq. (1). In Fig. 1 


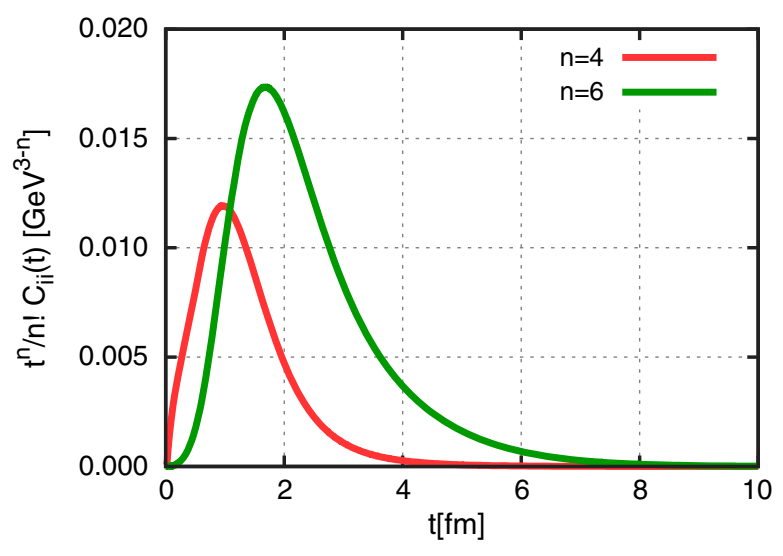

FIG. 1. Kernels of two moments of the spatial components of the zero three-momentum, electromagnetic current correlator as a function of Euclidean time [see Eq. (1) with $\mu=i=1,2$ or 3 and $\nu=0]$. As described in the text, these moments can be used to determine the slope and curvature of the HVP function at vanishing virtuality. The electromagnetic current correlator is obtained using a phenomenological description of the $e^{+} e^{-}$data compiled in [26] and dispersion relations, as briefly described in the text.

we plot the kernels of two such time-moments. They correspond to those needed for the first two derivatives of the HVP function at vanishing virtuality (the higher derivatives are obtained from higher moments). As the figure shows, the distances that need to be reached to reliably determine the slope and curvature are above $\sim 2$ and $\sim 4 \mathrm{fm}$, respectively. Higher derivatives require even larger distances. Here we address this challenge by performing a high-precision calculation on lattices of spatial extent $L \gtrsim 6 \mathrm{fm}$ and of time extent $T$, up to $11 \mathrm{fm}$. This calculation yields a prediction of QCD for these quantities that can be compared to present [31] and future phenomenological determinations. We leave to a forthcoming paper the computation of the anomalous magnetic moment of the muon.

\section{SIMULATIONS}

We employ a tree-level improved Symanzik gauge action [32] and a fermion action for four flavors of stout-smeared [33], staggered quarks. The up and down quark masses are treated as degenerate, their ratio to the strange quark mass is tuned to the vicinity of the physical point, which is defined from the Goldstone pion and kaon masses. The charm quark mass is fixed in units of the strange mass to $m_{c} / m_{s}=$ 11.85 [34]. When working with staggered fermions, a charged pion comes in sixteen "tastes," of which the "Goldstone" is the lightest. In the continuum limit the fifteen taste partners become degenerate with the Goldstone and continuum QCD is recovered through a "rooting" procedure [35]. At finite lattice spacing, the Golstone's taste partners are more massive. For our finest lattice spacing the root mean squared pion mass is about $15 \%$ larger than the Goldstone pion mass.

To set the physical mass point we use the isospin corrected pion and kaon masses, $M_{\pi}=134.8 \mathrm{MeV}$ and $M_{K}=494.2 \mathrm{MeV}$, from [36], as well as the electromagnetically corrected $\eta_{c}$ mass, $M_{\eta_{c}}=2.9863(27) \mathrm{GeV}$ of [37]. To convert the lattice results into physical units, we use the pion decay constant $f_{\pi}=130.50(1)(3)(13) \mathrm{MeV}$ [26] which is free of electromagnetic corrections and, to very good accuracy, equals to the decay constant in the $m_{d}=m_{u}$ limit [38]. This makes our definition of the physical point well defined in the isospin limit. In intermediate steps of the analysis we use the Wilson-flow-based [39] $w_{0}$-scale [40].

Table I lists the approximate lattice spacing, the lattice dimensions and the number of configurations used for computing the various quark-connected and disconnected contributions, for each of the six values of the bare coupling $\beta$ considered here. At each value of $\beta$, up to four independent simulations were performed, with slightly different values of the bare quark masses, so as to bracket the physical mass point. This is shown in Fig. 2, where the location of each simulation is plotted in an equivalent of the $m_{u d}-\left(m_{s} / m_{u d}\right)$ plane, where $m_{u d}$ and $m_{s}$ are, respectively, the average $u-d$ and $s$ masses.

A configuration corresponds to 10 unit length rational hybrid Monte Carlo (RHMC) [41] trajectories. The integration over the trajectory is improved with the gradient of the RHMC force $[42,43]$. On each trajectory we determine the topological charge, using a gauge field definition, after evolving the gauge fields with the Wilson flow up to a flow time $t=\omega_{0}^{2}$. This definition allowed a reliable determination of the topological susceptibility in [44]. On our finest lattices, we find that this charge has an integrated autocorrelation time of 22(4) trajectories, i.e. roughly two of our configurations, as determined according to the procedure of [45]. Since we bin all observables studied here in groups of at least four configurations, our statistical errors should be fully reliable. More information on the action together with simulation and algorithmic details can be found in [46].

TABLE I. List of $\beta$, lattice spacings, sizes and number of configurations used for the connected and disconnected correlators.

\begin{tabular}{lcccc}
\hline \hline$\beta$ & $a[\mathrm{fm}]$ & $T \times L$ & \#conf-conn & \#conf-disc \\
\hline 3.7000 & 0.134 & $64 \times 48$ & 1000 & 1000 \\
3.7500 & 0.118 & $96 \times 56$ & 1500 & 1500 \\
3.7753 & 0.111 & $84 \times 56$ & 1500 & 1500 \\
3.8400 & 0.095 & $96 \times 64$ & 2500 & 1500 \\
3.9200 & 0.078 & $128 \times 80$ & 3500 & 1000 \\
4.0126 & 0.064 & $144 \times 96$ & 450 & \\
\hline \hline
\end{tabular}




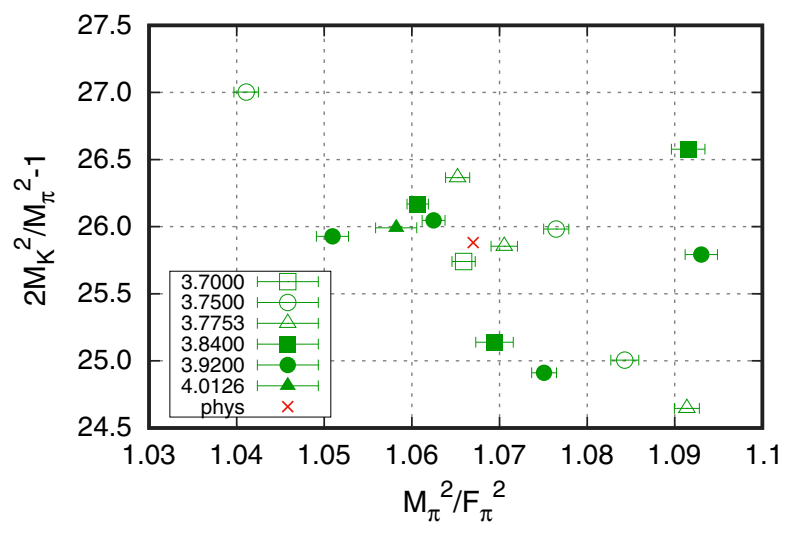

FIG. 2. Location of our 15 simulations in the $\left(M_{\pi} / F_{\pi}\right)^{2}-$ $2 M_{K}^{2} / M_{\pi}^{2}-1$ plane, compared to that of the physical mass point described in the text. Error bars in the vertical direction are smaller than the symbols. To leading order in chiral perturbation theory $(\chi \mathrm{PT}),\left(M_{\pi} / F_{\pi}\right)^{2}$ is proportional to $m_{u d}$ and $2 M_{K}^{2} / M_{\pi}^{2}-1$ is equal to $m_{s} / m_{u d}$. Different symbols correspond to different $\beta$ and thus lattice spacings.

\section{OBSERVABLES}

The hadronic vacuum polarization is derived from the electromagnetic current $j_{\mu}$, which is defined as $j_{\mu} / e=$ $\frac{2}{3} \bar{u} \gamma_{\mu} u-\frac{1}{3} \bar{d} \gamma_{\mu} d-\frac{1}{3} \bar{s} \gamma_{\mu} s+\frac{2}{3} \bar{c} \gamma_{\mu} c$, where $e$ is the unit of electromagnetic charge. From this we build the currentcurrent correlator $\left\langle j_{\mu}(x) j_{\nu}(0)\right\rangle$, in which we use the conserved lattice current at the source and sink. No renormalization is therefore necessary.

We split up the correlator in two different ways. First $\left\langle j_{\mu} j_{\nu}\right\rangle=\frac{e^{2}}{9}\left(5 C_{\mu \nu}^{l}+C_{\mu \nu}^{s}+4 C_{\mu \nu}^{c}+C_{\mu \nu}^{\mathrm{disc}}\right)$, where the first three terms contain the connected contractions for the light, strange and charm flavors, and the last contains the disconnected contractions. Flavor mixing terms arise only in the latter. We can also separate the correlator according to isospin symmetry, which is exact in our simulations: $\left\langle j_{\mu} j_{\nu}\right\rangle=\left\langle j_{\mu} j_{\nu}\right\rangle_{I=0}+\left\langle j_{\mu} j_{\nu}\right\rangle_{I=1}$. Here the isospin singlet contribution is $\left\langle j_{\mu} j_{\nu}\right\rangle_{I=0}=\frac{e^{2}}{18}\left(C_{\mu \nu}^{l}+2 C_{\mu \nu}^{s}+\right.$ $\left.8 C_{\mu \nu}^{c}+2 C_{\mu \nu}^{\text {disc }}\right)$, whereas the isospin triplet one is $\left\langle j_{\mu} j_{\nu}\right\rangle_{I=1}=\frac{e^{2}}{2} C_{\mu \nu}^{l}$. The lowest energy state contains three/two pions in the isospin singlet/triplet channel. This fact determines the behavior of the correlator for large separations.

We calculate the connected contributions to the correlators using point sources. We use the all-mode-averaging technique (AMA) of [47] and 768 random source positions on each configuration for the light quarks, 64 sources for the strange and 4 for the charm. To compute the quarkdisconnected contributions, we apply AMA again, and exploit the approximate $\mathrm{SU}(3)$ flavor symmetry on around 6000 stochastic sources $[18,48]$. We use random fourvolume sources and compute the zero-momentum, time propagators, correcting for bias. For the disconnected contribution of the charm we apply a hopping parameter expansion. The computer time required for this entire analysis is of the same order as the time needed for the generation of configurations. The use of AMA allows us to achieve the same precision on the final observables as would be obtained using only high-precision inversions, for one-half to one-third the computer time.

The $n$th coefficient of a Taylor expansion of the vacuum polarization scalar, $\Pi\left(Q^{2}\right)$, around $Q^{2}=0$ (i.e. $\left[\partial^{n} \Pi\left(Q^{2}\right) /\right.$ $\left.\left.\left(\partial Q^{2}\right)^{n}\right]_{Q^{2}=0} / n !\right)$ can be decomposed as $\Pi_{n}=\frac{1}{9}\left(5 \Pi_{n}^{l}+\right.$ $\left.\Pi_{n}^{s}+4 \Pi_{n}^{c}+\Pi_{n}^{\text {disc }}\right)$, where each term is related to the respective, configuration-space correlator through moments [16,49]

$$
\Pi_{n, \mu \nu}^{f}=(-)^{n+1} \sum_{x} \frac{\hat{x}_{\nu}^{2 n+2}}{(2 n+2) !} C_{\mu \mu}^{f}(x)
$$

for $f=\{l, s, c$, disc $\}$, with $\nu \neq \mu$ and $\hat{x}$ defined as $\hat{x}_{\nu}=$ $\min \left(x_{\nu}, L_{\nu}-x_{\nu}\right)$ and where $L_{\mu}$ is the size of the lattice in the $\mu$-direction. In general the result depends on the choice of $\mu$ and $\nu$. Three different averages, which are invariant under spatial cubic transformations, can be constructed: one which is an average over spatial moments, $\nu=1,2,3$, of correlators of spatial currents, $\mu=1,2,3 \neq \nu$; another, an average over spatial moments of correlators of timelike currents, $\mu=4$; a third, an average over time moments, $\nu=4$, of correlators of spatial currents. We call these averages $\Pi_{n, s s}, \Pi_{n, 4 s}$ and $\Pi_{n, s 4}$, respectively. In the disconnected case we only have the $\Pi_{n, s 4}$ average. The averages $\Pi_{n, s s}, \Pi_{n, 4 s}$ and $\Pi_{n, s 4}$ can be different, which is a consequence of the finite lattice size and the asymmetry $T \neq L$.

In the case of the light and disconnected correlators the signal deteriorates quickly with increasing distance. In our analysis we consider the spatial moments, i.e. $s s$ and $4 s$, up to their full extent $(L / 2 \gtrsim 3.1 \mathrm{fm})$. For the time-moment sums, $s 4$, which can extend up to $T / 2 \lesssim 5.7 \mathrm{fm}$, we introduce a cut $t_{c}$ in time. For times greater than $t_{c}$ we replace the correlator by an upper and a lower bound. ${ }^{1}$ The connected light correlator is proportional to the isospin triplet one, whose lowest-energy contribution comes from a two-pion state. Therefore up to exponentially suppressed corrections in $T$ the correlator satisfies

$$
0 \leq C^{l}(t) \leq C^{l}\left(t_{c}\right) \frac{\varphi(t)}{\varphi\left(t_{c}\right)}
$$

where $\varphi(t)=\cosh \left[E_{2 \pi}(T / 2-t)\right]$ and $E_{2 \pi}$ is the energy of two pions, each with the smallest nonvanishing lattice momentum, for which we use $2 \pi / L$. Typically the two bounds agree for $t_{c} \gtrsim 3 \mathrm{fm}$, as seen for example on the top plot of Fig. 3 for $\Pi_{1, s 4}^{l}$ and of Fig. 4 for $\Pi_{2, s 4}^{l}$. These plots

\footnotetext{
${ }^{1}$ We are grateful to Christoph Lehner for a discussion of this issue.
} 


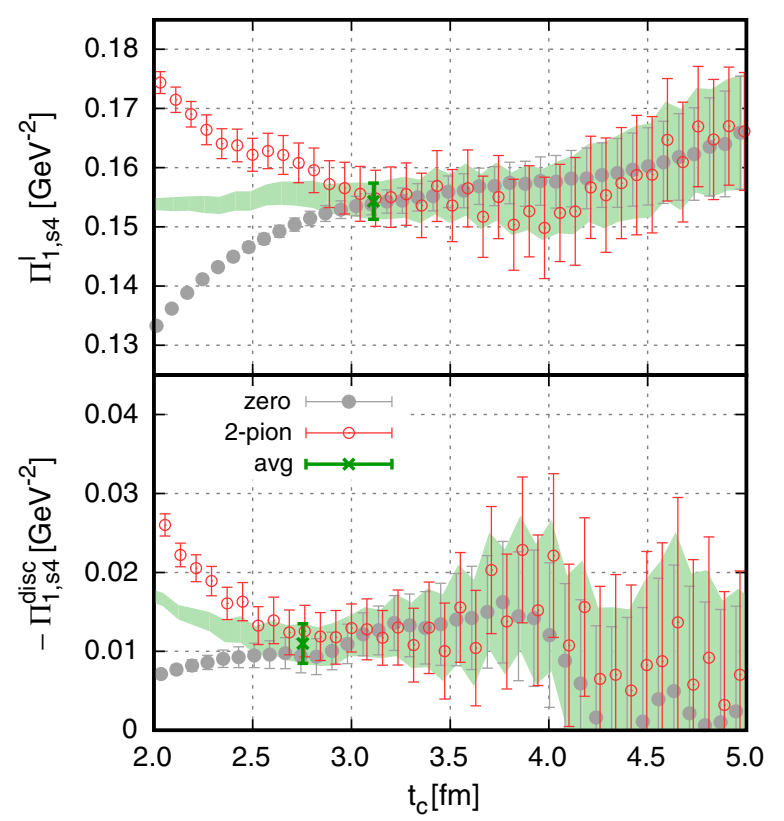

FIG. 3. Top: an upper/lower bound on the time moment $\Pi_{1, s 4}^{l}$ is obtained by setting the timelike correlator to have a two-pion decay/a value of zero, starting at the first lattice time beyond $t_{c}$. The open circles correspond to the upper bound, the filled circles, to the lower bound and the green band to the average of the two with errors. The cross with error bars is the average value which serves as our result $\Pi_{1, s 4}^{l}$ on this particular ensemble. Bottom: upper/lower bound on the time moment $\Pi_{1, s 4}^{\text {disc }}$ is obtained by replacing the correlator with zero/with the connected correlator that is given a two-pion decay rate, as explained in the text. The points and region follow the same nomenclature as in the top plot. Results are for an ensemble at $\beta=3.9200$.

also show the average of the two bounds which remains constant in a large region around $3 \mathrm{fm}$, until the signal degrades at larger $t_{c}$. In our analyses, we take $t_{c}=3.1 \mathrm{fm}$ on the light connected timelike correlators and consider the average of the two bounds, whose error covers the bounds' values, to get the final result. This result is depicted as a cross in these plots. Our approach allows us to control statistical errors without biasing the result. Pion-pion interactions can change the smallest two-pion momentum from $2 \pi / L$ in that channel. Using the model of [50] and neglecting four-pion contributions, we determine the change in the momentum to be around $3 \%$. We checked, that such a reduction of the momentum changes the result on $\Pi^{l}$ by a small fraction of the statistical error.

The disconnected contribution alone can be constrained for large enough time separations, where the isospin singlet channel, dominated by three-pion states, can be neglected compared to the triplet, dominated by two-pion ones. Here we have

$$
0 \geq\left[2 C^{s}+8 C^{c}+2 C^{\mathrm{disc}}\right](t) \geq-C^{l}\left(t_{c}\right) \frac{\varphi(t)}{\varphi\left(t_{c}\right)}
$$

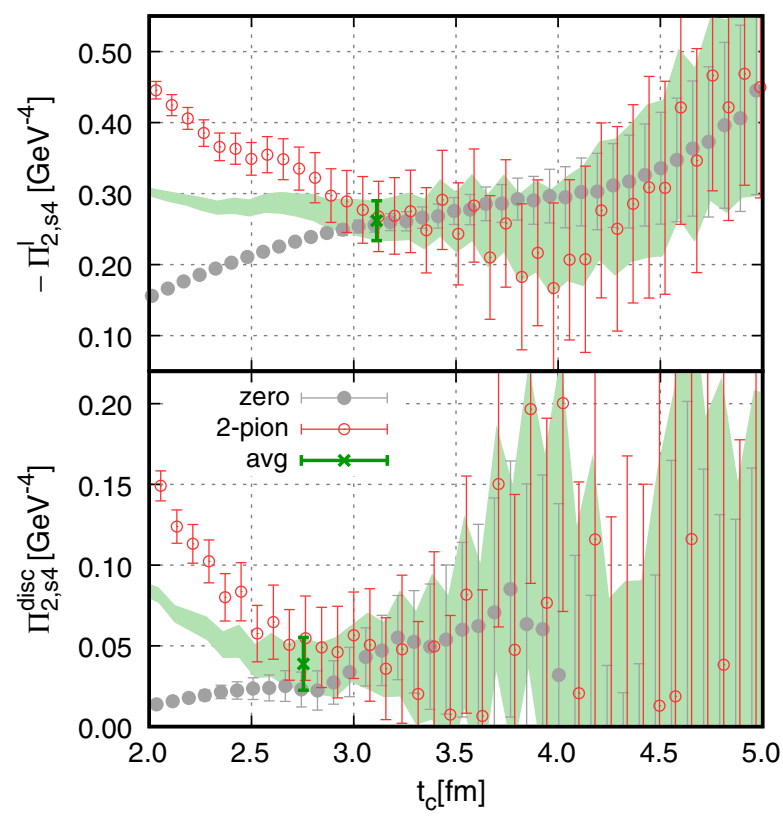

FIG. 4. Same as Fig. 3 but for $\Pi_{2, s 4}^{f}, f=l$, disc.

up to corrections exponentially suppressed in $T$. This gives an upper and a lower bound on $\Pi^{s}+4 \Pi^{c}+\Pi^{\text {disc }}$, which can be used to determine the time $t_{c}$ after which the two bounds agree within errors. At large $t$, the connected strange and charm contributions in (3) are exponentially suppressed, and their presence does not make a difference when determining $t_{c}$ so we neglect them. In the bottom plots of Fig. 3 and Fig. 4, we show the upper and lower bounds on $\Pi_{1, s 4}^{\text {disc }}$ and $\Pi_{2, s 4}^{\text {disc }}$, respectively. In our analyses we take as a final value the average of the two bounds considering $t_{c}=2.7 \mathrm{fm}$.

\section{RESULTS}

To obtain our final results in the continuum limit and at the physical point, we fit the lattice results to functions which depend on the pion, kaon and $\eta_{c}$ masses and on the lattice spacing squared $a^{2}$. Since the simulations were done around the physical point, a linear pion/kaon mass squared and $\eta_{c}$ mass dependence is always sufficient. For all contributions reasonable fit qualities can be achieved with a linear $a^{2}$ dependence.

We begin with the connected, light-quark contribution $\Pi^{l}$. Here, a dependence linear in $M_{\pi}^{2}, M_{K}^{2}$ and $a^{2}$ is sufficient. We pay special attention to the difference between the averages $\Pi_{s s}^{l}, \Pi_{4 s}^{l}$ and $\Pi_{s 4}^{l}$. Some ensembles show differences, but these are not significant statistically. If we assume no difference between the three averages and fit all ensembles and all three averages together in one fit, we get a reasonable fit quality $\chi^{2} /$ dof $=45 / 41$. Also, if we include additional fit parameters describing the difference between the three averages, they come out zero within error bars. This remains true after dropping the coarser lattices 
from the fit, for which finite-volume effects are reduced by taste violations. Indeed, for coarser lattices, the tasteaveraged pion mass is larger, thus reducing the dominant, two-pion contribution to these effects. Therefore we average over the combinations $s s, 4 s$ and $s 4$ in this work and leave an investigation of differences for the future, when statistical errors are reduced. The upper panel of Fig. 5 shows the continuum extrapolation of $\Pi_{1}^{l}$. The coarsest lattice gives a value about $20 \%$ smaller than the continuum limit. For $\Pi_{2}^{l}$, the difference is around $35 \%$, as shown in Fig. 6. The final central value for the continuum limit is the

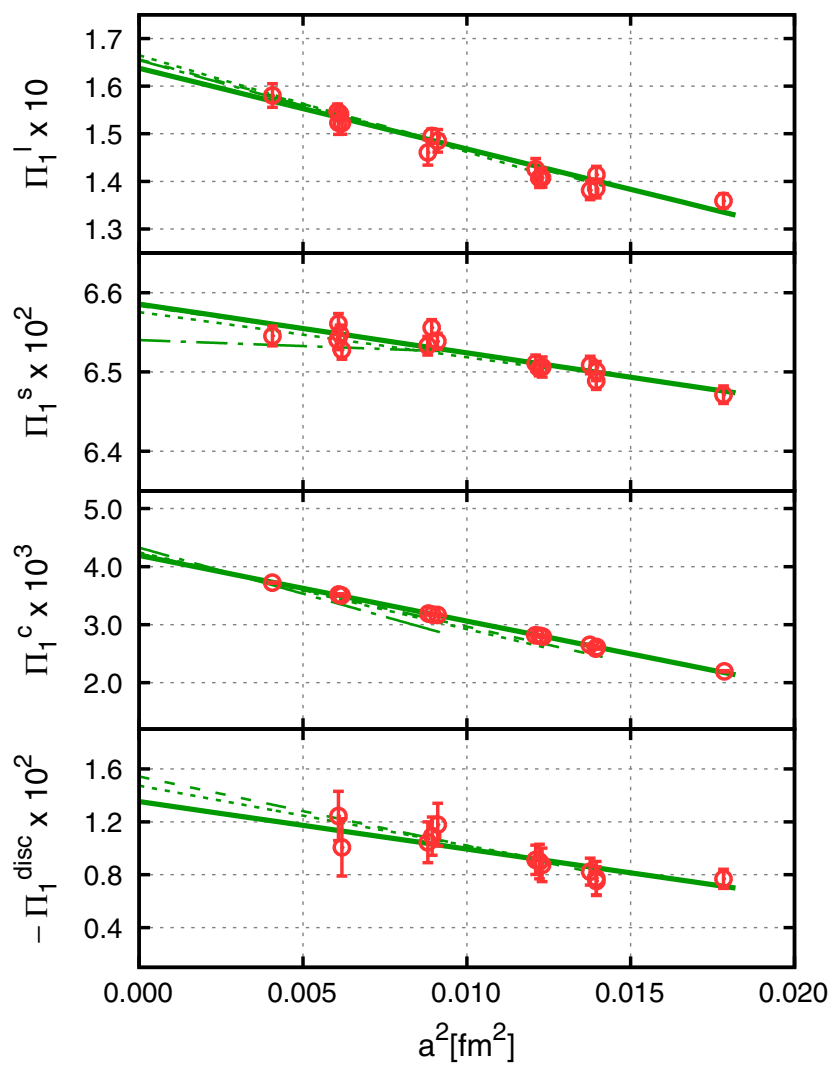

FIG. 5. Continuum extrapolations of the light, strange, charm and disconnected contributions to $\Pi_{1}$, in units of $\mathrm{GeV}^{-2}$. The lines are fits linear in $a^{2}$ corresponding to the different cuts in lattice spacing described in the text. The solid lines corresponds to including all lattices spacings; the dashed lines to omitting the coarsest simulation; the dotted lines to omitting the simulations corresponding to the two coarsest lattices spacings; the dotdashed lines to omitting those associated with the three largest values of $a$. The red open circles with errors are the results from our 15 simulations for the light and strange, 13 for the charm and 12 for the disconnected contributions, with statistical uncertainties. These points have been interpolated to the physical mass point using the fits that include all lattice spacings (solid lines). Thus, the fact that some of the other lines do not go perfectly through these points does not mean that the corresponding fits are bad. Rather, it indicates that the dependence on the other lattice parameters in those fits is slightly different from the one corresponding to the solid-line fit.

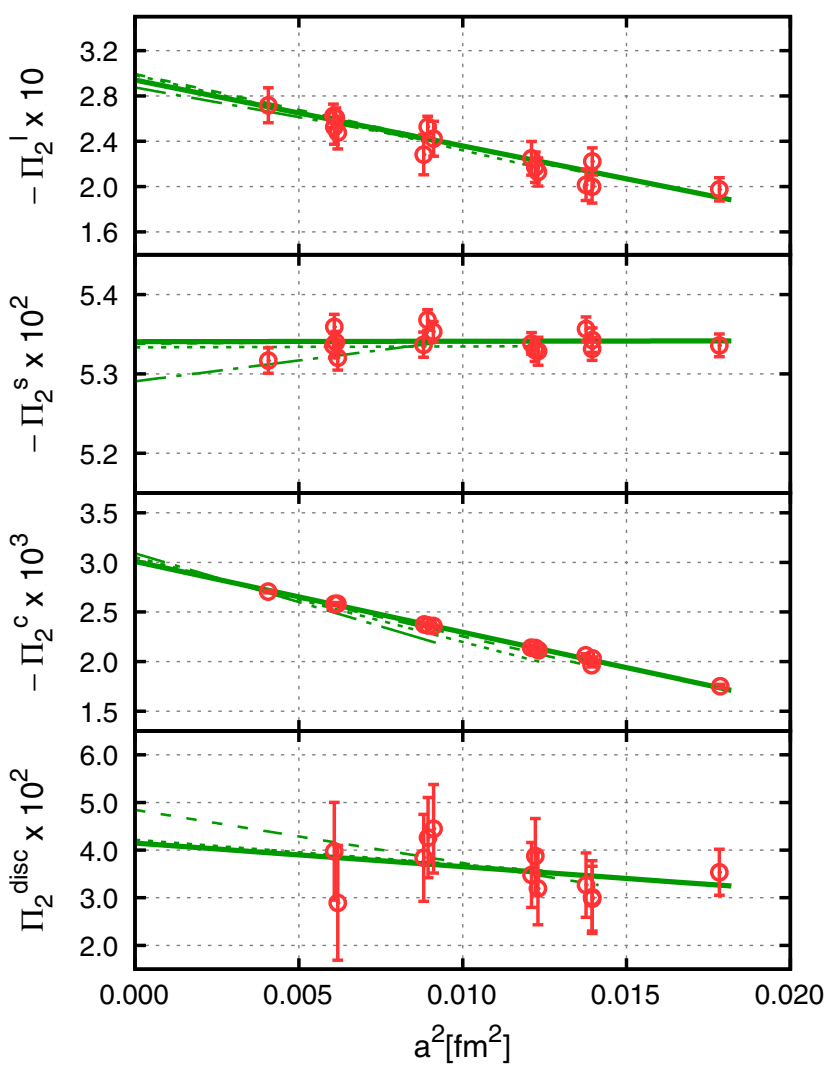

FIG. 6. Same as Fig. 5 but for the light, strange, charm and disconnected contributions to $\Pi_{2}$, in units of $\mathrm{GeV}^{-4}$.

mean of the uniformly-weighted distribution obtained by imposing four different cuts on lattice spacing (no cut, $a \leq 0.118,0.111,0.095 \mathrm{fm})$ in the extended frequentist approach of $[51,52]$. The systematic error is chosen to cover the central values of all continuum extrapolations. The results for the light-quark contribution to the slope and curvature are given in Table II.

The connected, strange and charm quark contributions are plotted in the second and third panels of Figs. 5-6 and the respective continuum extrapolated values are given in Table II. For the strange contribution, a linear dependence on $M_{\pi}^{2}, M_{K}^{2}$ and $a^{2}$ describes the data well. An additional dependence on $M_{\eta_{c}}$ is needed for the charmed channel to correct a slight mistuning of the charm-quark mass. The strange channel has much smaller lattice artifacts than the light, since it is much less affected by taste violations. The difference between the continuum and the coarsest lattice is about $2 \%$ for $\Pi_{1}^{s}$ and about $0.1 \%$ for $\Pi_{2}^{s}$. For the charm the fit qualities are worse, because the precision of the data is orders of magnitude better than for the lighter flavors. Here we fit only a subset of 40 configurations, maximally spaced along the simulation Markov chain. This increases the statistical error and leads to good fit qualities. Results on the coarsest lattice deviate by about $50 \%$ from the continuum limit for $\Pi_{1}^{c}$ and $40 \%$ for $\Pi_{2}^{c}$. 
TABLE II. Final results on the first and second derivative of the hadronic vacuum polarization scalar at zero squared-momentum. The first four lines contain the continuum extrapolated lattice results. The next three lines are the isospin singlet, triplet and total contributions. The first error is statistical and the second is systematic, as determined directly from simulation results. The latter is dominated by discretization uncertainties. In the following line we give an estimate of the finite-volume effects of the $I=1$ contribution using LO $\chi \mathrm{PT}$. In that approach, the finite-volume effects on $\Pi^{l} / \Pi^{\text {disc }}$ are $2 /-1$ times those on the $I=1$ contribution, whereas those on the $I=0$ contribution vanish. The last line contains the total contribution corrected for finite-volume and isospin breaking effects, the latter estimated using phenomenology, as described in the text. In these results, the third uncertainty is the one associated with the finite-volume correction and the fourth, with isospin breaking effects.

\begin{tabular}{lcc}
\hline \hline & $\Pi_{1}\left[\mathrm{GeV}^{-2}\right]$ & $\Pi_{2}\left[\mathrm{GeV}^{-4}\right]$ \\
\hline Light & $0.1653(17)(16)$ & $-0.295(10)(7)$ \\
Strange $\times 10^{2}$ & $6.57(1)(3)$ & $-5.33(1)(4)$ \\
Charm $\times 10^{4}$ & $42.5(2)(8)$ & $-3.04(4)(5)$ \\
Disconnected $\times 10^{2}$ & $-1.5(2)(1)$ & $4.4(1.0)(0.4)$ \\
$I=0$ & $0.0168(2)(2)$ & $-0.018(1)(1)$ \\
$I=1$ & $0.0827(8)(8)$ & $-0.147(5)(4)$ \\
Total & $0.0994(10)(9)$ & $-0.165(6)(4)$ \\
$I=1$ FV correction & $0.0006(23)$ & $-0.016(10)$ \\
Total + FV + I B & $\mathbf{0 . 1 0 0 0}(\mathbf{1 0})(\mathbf{9})(\mathbf{2 3})(\mathbf{1 3})$ & $\mathbf{0 . 1 8 1}(\mathbf{6})(\mathbf{4})(\mathbf{1 0})(\mathbf{2})$ \\
\hline \hline
\end{tabular}

The lattice spacing dependence of the disconnected $\Pi_{1}$ and $\Pi_{2}$ are shown in the bottom panels of Figs. 5-6. We calculated the charm quark contribution to the disconnected term on the coarsest lattice: we found it to be less than about $1 \%$ of the total disconnected result for both $\Pi_{1}$ and $\Pi_{2}$. Assuming that the continuum extrapolation does not change the order of magnitude of the relative size of the contributions, this means that the charm quark contribution to $\Pi_{1}^{\text {disc }}$ is more than ten times smaller than the statistical error on the total disconnected contribution, sixty times smaller than that on the total $\Pi_{1}$, and smaller still for $\Pi_{2}$. We can therefore safely discard the charm from the disconnected term at all lattice spacings. As Figs. 5-6 show, $\Pi_{1}^{\text {disc }}$ on the coarsest lattice, deviates by about $50 \%$ from the continuum limit while this figure for $\Pi_{2}^{\text {disc }}$ is around $20 \%$. Since we have one less lattice spacing available than in the connected cases, we apply only three cuts in $\beta$. Our results for these disconnected contributions are summarized in Table II.

The total result for $\Pi_{1}$ is the sum of the four contributions, with weights given in the second paragraph of Sec. III. It has a combined statistical and continuum extrapolation error of $1.4 \%$. All of these results apply to a box size of $\sim 6 \mathrm{fm}$ and to the isospin symmetric case.

In the absence of a systematic study with simulations in a variety of volumes, only model estimates of finite-volume effects can be made. As argued in [53,54], for large volumes those effects will be governed by pion contributions that can be computed in $\chi \mathrm{PT}[53]$. Since the $I=0$ channel is dominated by three-pion exchange, the finitesize effects are expected to be smaller than those of the $I=1$ contribution, which are already small. Thus we consider only the latter. For all three index combinations, $s s, 4 s$ and $s 4$, we calculate the difference between the infinite and finite volume moments at one loop in $\chi \mathrm{PT}$. We then take the average of the maximum and the minimum differences as our central value, with an uncertainty given by the half distance between the maximum and minimum. We record these corrections for $\Pi_{1}$ and $\Pi_{2}$ in Table II. For the first derivative the correction is on the level of $2 \%$, whereas on the second derivative it is of order $10 \%$. This correction increases rapidly with moment number, therefore we have chosen not to quote moments beyond the second one.

Concerning isospin breaking corrections, while little is known about how they modify the slope and curvature of the hadronic vacuum polarization function, more is known about their contribution to the anomalous magnetic moment of the muon. Compared to phenomenological determinations [4-8], our $m_{d}=m_{u}$ calculation without QED is missing a number of effects ${ }^{2}$ Noting their contributions to $a_{\mu}^{\mathrm{LO}-\mathrm{HVP}}$ in parentheses in units of $10^{-10}$, these effects are $\rho-\omega(2.80 \pm 0.19$ from [55]) and $\rho-\gamma$ $(-2.71 \pm 0.27$ from $[56,57])$ mixing, final state radiation $(3.86 \pm 0.39$ from [2] with a $10 \%$ error added), and the $\pi^{0} \gamma \quad(4.42 \pm 0.19$ from [5]) and $\eta \gamma \quad(0.64 \pm 0.02$ from [5]) contributions. This leads to a correction of $(9.0 \pm 0.5) \times 10^{-10}$, i.e. $1.3 \%$ of the result for $a_{\mu}^{\mathrm{LO}-\mathrm{HVP}}$ given in [5]. However, a competing effect enters. In our calculation without electromagnetism, the charged pion has a mass which is smaller than its physical value (see above).

\footnotetext{
${ }^{2}$ We are grateful to Maurice Benayoun and Fred Jegerlehner for very informative discussions on this subject.
} 
But a smaller charged-pion mass has the effect of enhancing the two-pion contribution to $a_{\mu}^{\mathrm{LO}-\mathrm{HVP}}$ and thus leads to a correction whose sign is opposite to the correction associated with the sum of effects discussed above. Moreover, a phenomenological description based on $e^{+} e^{-}$data indicates that their magnitudes are very close [57]. Thus, we assume here that the total correction which has to be added to an isospin-limit determination of $a_{\mu}^{\mathrm{LO}-\mathrm{HVP}}$ is $(0.0 \pm 1.3) \%$, where we have taken the error to be of the typical size of the corrections themselves. Because of the dominant role which $\Pi_{1}$ plays in determining $a_{\mu}^{\mathrm{LO}-\mathrm{HVP}}$, one expects a tantamount correction on that coefficient. Inferring the correction on $\Pi_{2}$ is less direct, but we assume here that it is of the same size as for $\Pi_{1}$. Thus, we add $(0.0 \pm 1.3) \%$ of $\Pi_{1}$ and $\Pi_{2}$ to our results for these quantities, after they have been corrected for finite-volume effects.

Putting everything together we quote our final results for the first two moments in the last row of Table II. Combining all four errors in quadrature, we obtain $\Pi_{1}$ with a total uncertainty of $3.0 \%$ and $\Pi_{2}$ of $6.9 \%$.

\section{DISCUSSION AND CONCLUSIONS}

It is interesting to compare these results with those in the literature. The only other lattice determinations of $\Pi_{1}$ and $\Pi_{2}$, near the physical mass point, are obtained from two, $N_{f}=2+1+1$ staggered simulations with coarse lattice spacings $a=0.12$ and $0.15 \mathrm{fm}$ [58]. However, since the action used is different from ours and a comparison with our continuum limit results could be misleading, we choose not to exhibit such a comparison here.

The only phenomenological determination of the slope and derivative parameters, $\Pi_{1}$ and $\Pi_{2}$, is the recent one of [31]. Taking their "data direct" results, which are obtained from an interpolation of $e^{+} e^{-} \rightarrow$ hadrons data, and converting them to our conventions, we get $\Pi_{1}=$ 0.0990(7) $\mathrm{GeV}^{-2}$ and $\Pi_{2}=-0.2057(16) \mathrm{GeV}^{-4}$. These numbers can be compared to our final results, i.e. those given in the last row of Table II. In absolute value, their result for $\Pi_{1}$ is 0.3 combined standard deviations smaller than ours and for $\Pi_{2}, 1.9 \sigma$ larger. The latter might be due to an underestimate of $\mathrm{FV}$ corrections in our determination of the second moment, or some problem with the experimental data used in the phenomenological analysis of [31].

As mentioned in the Introduction, we leave a careful determination of the LO HVP contribution to the anomalous magnetic moment of the muon to a future publication. However, to give a sense of what our results mean in terms of this quantity, we naively determine it using the $[1,1]$ Padé discussed in the Introduction. We find a value of around $690 \times 10^{-10}$ with, roughly, a $3 \%$ uncertainty coming from the uncertainties on our determination of $\Pi_{1}$ and $\Pi_{2}$ and an additional $2 \%$ coming from the use of a $[1,1]$ Padé. This estimate is compatible with recent phenomenological determinations [6-8], though much less precise, as well as somewhat larger, but still compatible within errors, with the only other two $N_{f}=2+1+1$ lattice results $[14,19]$.

\section{ACKNOWLEDGMENTS}

L. L. thanks M. Benayoun, C. Davies, F. Jegerlehner, C. Lehner, H. Leutwyler and E. de Rafael for very informative discussions. Computations were performed on JUQUEEN and JUROPA at Forschungszentrum Jülich, on Turing at the Institute for Development and Resources in Intensive Scientific Computing (IDRIS) in Orsay, on SuperMUC at Leibniz Supercomputing Centre in München, on Hermit at the High Performance Computing Center in Stuttgart. This project was supported by in part by the OCEVU Laboratoire d'excellence (ANR-11-LABX-0060) and the A*MIDEX Project (ANR-11-IDEX-0001-02), which are funded by the "Investissements d'avenir" French government program and managed by the "Agence nationale de la recherche" (ANR), by the DFG grant SFB/TR55, by the Gauss Centre for Supercomputing e.V and by the GENCI-IDRIS supercomputing Grant No. 52275. R. M. was supported in part by a joint Ph.D. fellowship from the Centre national de la recherche scientifique (CNRS) and the Commissariat à l'énergie atomique et aux énergies alternatives (CEA).
[1] F. Jegerlehner, Nucl. Phys. B, Proc. Suppl. 181-182, 135 (2008).

[2] F. Jegerlehner and A. Nyffeler, Phys. Rep. 477, 1 (2009).

[3] J. P. Miller, E. de Rafael, B. L. Roberts, and D. Stöckinger, Annu. Rev. Nucl. Part. Sci. 62, 237 (2012).

[4] S. Eidelman and F. Jegerlehner, Z. Phys. C 67, 585 (1995).

[5] M. Davier, A. Hoecker, B. Malaescu, and Z. Zhang, Eur. Phys. J. C 71, 1515 (2011); 72, 1874(E) (2012).
[6] K. Hagiwara, R. Liao, A. D. Martin, D. Nomura, and T. Teubner, J. Phys. G 38, 085003 (2011).

[7] M. Davier, Nucl. Part. Phys. Proc. 287-288, 70 (2017).

[8] F. Jegerlehner, arXiv:1705.00263.

[9] G. W. Bennett et al. (Muon g-2 Collaboration), Phys. Rev. D 73, 072003 (2006).

[10] T. Blum, Phys. Rev. Lett. 91, 052001 (2003).

[11] C. Aubin and T. Blum, Phys. Rev. D 75, 114502 (2007). 
[12] X. Feng, K. Jansen, M. Petschlies, and D. B. Renner, Phys. Rev. Lett. 107, 081802 (2011).

[13] M. Della Morte, B. Jager, A. Juttner, and H. Wittig, J. High Energy Phys. 03 (2012) 055.

[14] F. Burger, X. Feng, G. Hotzel, K. Jansen, M. Petschlies, and D. B. Renner, J. High Energy Phys. 02 (2014) 099.

[15] T. Blum, M. Hayakawa, and T. Izubuchi, Proc. Sci., LATTICE2012 (2012) 022, [arXiv:1301.2607].

[16] B. Chakraborty, C. T. H. Davies, G. C. Donald, R. J. Dowdall, J. Koponen, G. P. Lepage, and T. Teubner, Phys. Rev. D 89, 114501 (2014).

[17] G. S. Bali, F. Bruckmann, G. Endrödi, S. D. Katz, and A. Schäfer, J. High Energy Phys. 08 (2014) 177.

[18] T. Blum, P. A. Boyle, T. Izubuchi, L. Jin, A. Jüttner, C. Lehner, K. Maltman, M. Marinkovic, A. Portelli, and M. Spraggs, Phys. Rev. Lett. 116, 232002 (2016).

[19] B. Chakraborty, C. T. H. Davies, J. Koponen, G. P. Lepage, M. J. Peardon, and S. M. Ryan, Phys. Rev. D 93, 074509 (2016).

[20] T. Blum et al. (RBC/UKQCD Collaboration), J. High Energy Phys. 04 (2016) 063; 05 (2017) 034(E).

[21] M. Della Morte, A. Francis, V. Gülpers, G. Herdoíza, G. von Hippel, H. Horch, B. Jäger, H. B. Meyer, A. Nyffeler, and H. Wittig, arXiv:1705.01775.

[22] J. L. Holzbauer, J. Phys. Conf. Ser. 770, 012038 (2016).

[23] M. Otani (E34 Collaboration), J. Phys. Soc. Jpn. Conf. Proc. 8, 025008 (2015).

[24] J. S. Bell and E. de Rafael, Nucl. Phys. B11, 611 (1969).

[25] E. de Rafael, Phys. Lett. B 736, 522 (2014).

[26] K. A. Olive et al. (Particle Data Group Collaboration), Chin. Phys. C 38, 090001 (2014).

[27] C. Aubin, T. Blum, M. Golterman, and S. Peris, Phys. Rev. D 86, 054509 (2012).

[28] S. Borsanyi, Z. Fodor, T. Kawanai, S. Krieg, L. Lellouch, R. Malak, K. Miura, K. K. Szabo, C. Torrero, and B. Toth (Budapest-Marseille-Wuppertal Collaboration), arXiv:1612 $.02364 \mathrm{v} 1$.

[29] E. de Rafael, Phys. Rev. D 96, 014510 (2017).

[30] C. A. Dominguez, K. Schilcher, and H. Spiesberger, arXiv: 1704.02843

[31] M. Benayoun, P. David, L. DelBuono, and F. Jegerlehner, arXiv:1605.04474.

[32] M. Luscher and P. Weisz, Commun. Math. Phys. 97, 59 (1985); 98, 433(E) (1985).

[33] C. Morningstar and M. J. Peardon, Phys. Rev. D 69, 054501 (2004).

[34] C. T. H. Davies, C. McNeile, K. Y. Wong, E. Follana, R. Horgan, K. Hornbostel, G. P. Lepage, J. Shigemitsu, and H. Trottier, Phys. Rev. Lett. 104, 132003 (2010).
[35] C. Bernard, M. Golterman, Y. Shamir, and S. R. Sharpe, Phys. Rev. D 77, 114504 (2008).

[36] S. Aoki et al., Eur. Phys. J. C 77, 112 (2017).

[37] B. Chakraborty, C. T. H. Davies, B. Galloway, P. Knecht, J. Koponen, G. C. Donald, R. J. Dowdall, G. P. Lepage, and C. McNeile, Phys. Rev. D 91, 054508 (2015).

[38] J. Gasser and G. R. S. Zarnauskas, Phys. Lett. B 693, 122 (2010).

[39] M. Lüscher, J. High Energy Phys. 08 (2010) 071.

[40] S. Borsanyi et al. (Budapest-Marseille-Wuppertal Collaboration), J. High Energy Phys. 09 (2012) 010.

[41] M. A. Clark and A. D. Kennedy, Phys. Rev. Lett. 98, 051601 (2007).

[42] M. A. Clark, B. Joo, A. D. Kennedy, and P. J. Silva, Phys. Rev. D 84, 071502 (2011).

[43] H. Yin and R. D. Mawhinney, Proc. Sci., LATTICE2011 (2011) 051, [arXiv:1111.5059].

[44] S. Borsanyi et al., Nature (London) 539, 69 (2016).

[45] U. Wolff (ALPHA Collaboration), Comput. Phys. Commun. 156, 143 (2004); 176, 383(E) (2007).

[46] R. Bellwied, S. Borsanyi, Z. Fodor, S. D. Katz, A. Pasztor, C. Ratti, and K. K. Szabo, Phys. Rev. D 92, 114505 (2015).

[47] T. Blum, T. Izubuchi, and E. Shintani, Phys. Rev. D 88, 094503 (2013).

[48] A. Francis, V. Gülpers, B. Jäger, H. Meyer, G. von Hippel et al., Proc. Sci., LATTICE2014 (2014) 128, [arXiv:1411 .7592].

[49] X. Feng, S. Hashimoto, G. Hotzel, K. Jansen, M. Petschlies, and D. B. Renner, Phys. Rev. D 88, 034505 (2013).

[50] M. Lüscher, Nucl. Phys. B364, 237 (1991).

[51] S. Durr et al. (Budapest-Marseille-Wuppertal Collaboration), Science 322, 1224 (2008).

[52] S. Borsanyi et al. (Budapest-Marseille-Wuppertal Collaboration), Science 347, 1452 (2015).

[53] C. Aubin, T. Blum, P. Chau, M. Golterman, S. Peris, and C. Tu, Phys. Rev. D 93, 054508 (2016).

[54] A. Francis, B. Jaeger, H. B. Meyer, and H. Wittig, Phys. Rev. D 88, 054502 (2013).

[55] M. Davier, A. Hoecker, G. L. Castro, B. Malaescu, X. H. Mo, G. T. Sanchez, P. Wang, C. Z. Yuan, and Z. Zhang, Eur. Phys. J. C 66, 127 (2010).

[56] F. Jegerlehner and R. Szafron, Eur. Phys. J. C 71, 1632 (2011).

[57] M. Benayoun and F. Jegerlehner (private communication).

[58] B. Chakraborty, C. T. H. Davies, P. G. de Oliviera, J. Koponen, G. P. Lepage, and R. S. Van de Water, Phys. Rev. D 96, 034516 (2017). 